\title{
A class of retarded nonlinear integral inequalities and its application in nonlinear differential-integral equation
}

Wu-Sheng Wang*

\section{${ }^{*}$ Correspondence:}

wang4896@126.com

Department of Mathematics, Hechi

University, Yizhou, Guangxi 546300,

P.R. China

\begin{abstract}
In this paper, we discuss a class of retarded nonlinear integral inequalities and give an upper bound estimation of an unknown function by the integral inequality technique. This estimation can be used as a tool in the study of differential-integral equations with the initial conditions.

MSC: 26D10; 26D15; 26D20; 34A12; 34A40
\end{abstract}

Keywords: integral inequality; analysis technique; retarded differential-integral equation; estimation

\section{Introduction}

Gronwall-Bellman inequalities $[1,2]$ can be used as important tools in the study of existence, uniqueness, boundedness, stability, and other qualitative properties of solutions of differential equations, integral equations, and integral-differential equations. There can be found a lot of generalizations of Gronwall-Bellman inequalities in various cases from literature (e.g., [3-13]).

Lemma 1 (Abdeldaim and Yakout [4]) We assume that $u(t)$ and $f(t)$ are nonnegative realvalued continuous functions defined on $I=[0, \infty)$ and they satisfy the inequality

$$
u^{p+1}(t) \leq u_{0}+\left(\int_{0}^{t} f(s) u^{p}(s) d s\right)^{2}+2 \int_{0}^{t} f(s) u^{p}(s)\left[u^{p}(s)+\int_{0}^{s} f(\lambda) u^{p}(\lambda) d \lambda\right] d s
$$

for all $t \in I$, where $u_{0}>0$ and $p \in(0,1)$ are constants. Then

$$
u(t) \leq u_{0}^{\frac{1}{p+1}}+\frac{2}{p+1} \int_{0}^{t} f(s) D_{2}(s) d s, \quad \forall t \in I
$$

where

$$
D_{2}(t)=\beta(t)\left[u_{0}^{\frac{1-p}{1+p}}+2 \frac{1-p}{1+p} \int_{0}^{t} f(s) \exp \left(-2 \frac{1-p}{p} \int_{0}^{s} f(\lambda) d \lambda\right) d s\right]^{\frac{p}{1-p}}
$$

and $\beta(t)=\exp \left(2 \int_{0}^{t} f(s) d s\right)$, for all $t \in I$.

\section{Springer}

( 2012 Wang; licensee Springer. This is an Open Access article distributed under the terms of the Creative Commons Attribution License (http://creativecommons.org/licenses/by/2.0), which permits unrestricted use, distribution, and reproduction in any medium, provided the original work is properly cited. 
In this paper, we discuss a class of retarded nonlinear integral inequalities and give an upper bound estimation of an unknown function by the integral inequality technique.

\section{Main result}

In this section, we discuss some retarded integral inequalities of Gronwall-Bellman type. Throughout this paper, let $I=[0, \infty)$.

Theorem 1 Suppose $\alpha \in C^{1}(I, I)$ is increasing function with $\alpha(t) \leq t, \alpha(0)=0, \forall t \in I$. We assume that $u(t)$ and $f(t)$ are nonnegative real-valued continuous functions defined on $I$, and they satisfy the inequality

$$
\begin{aligned}
u^{p+1}(t) \leq & u_{0}+\left(\int_{0}^{\alpha(t)} f(s) u^{p}(s) d s\right)^{2} \\
& +2 \int_{0}^{\alpha(t)} f(s) u^{p}(s)\left[u^{p}(s)+\int_{0}^{s} f(\lambda) u^{p}(\lambda) d \lambda\right] d s, \quad \forall t \in I,
\end{aligned}
$$

where $u_{0}>0$ and $p \in(0,1)$ are constants. Then

$$
u(t) \leq u_{0}^{\frac{1}{p+1}}+\frac{2}{p+1} \int_{0}^{\alpha(t)} f(s) \theta_{1}\left(\alpha^{-1}(s)\right) d s, \quad \forall t \in I
$$

where

$$
\theta_{1}(t)=\beta_{1}(t)\left[u_{0}^{1-p}+2 \frac{1-p}{1+p} \int_{0}^{\alpha(t)} f(s) \exp \left(-2 \frac{1-p}{p} \int_{0}^{s} f(\lambda) d \lambda\right) d s\right]^{\frac{p}{1-p}},
$$

and $\beta_{1}(t)=\exp \left(2 \int_{0}^{\alpha(t)} f(s) d s\right)$, for all $t \in I$.

Remark 1 If $\alpha(t)=t$, then Theorem 1 reduces Lemma 1.

Proof Let $z_{1}^{p+1}(t)$ denote the function on the right-hand side of (2.1), which is a positive and nondecreasing function on $I$ with $z_{1}(0)=u_{0}^{\frac{1}{p+1}}$. Then (2.1) is equivalent to

$$
u(t) \leq z_{1}(t), \quad u(\alpha(t)) \leq z_{1}(\alpha(t)), \quad \forall t \in I .
$$

Differentiating $z_{1}^{p+1}(t)$ with respect to $t$, using (2.4) we have

$$
\begin{aligned}
(p+1) z_{1}^{p}(t) \frac{d z_{1}(t)}{d t}= & 2 \alpha^{\prime}(t) f(\alpha(t)) u^{p}(\alpha(t)) \int_{0}^{\alpha(t)} f(s) u^{p}(s) d s \\
& +2 \alpha^{\prime}(t) f(\alpha(t)) u^{p}(\alpha(t))\left[u^{p}(\alpha(t))+\int_{0}^{\alpha(t)} f(\lambda) u^{p}(\lambda) d \lambda\right] \\
\leq & 2 \alpha^{\prime}(t) f(\alpha(t)) z_{1}^{p}(t)\left[z_{1}^{p}(t)+2 \int_{0}^{\alpha(t)} f(\lambda) z_{1}^{p}(\lambda) d \lambda\right], \quad \forall t \in I .
\end{aligned}
$$

Since $z_{1}^{p}(t)>0$, from (2.5) we have

$$
\frac{d z_{1}(t)}{d t} \leq \frac{2}{p+1} \alpha^{\prime}(t) f(\alpha(t)) Y_{1}(t), \quad \forall t \in I
$$


where

$$
Y_{1}(t):=z_{1}^{p}(t)+2 \int_{0}^{\alpha(t)} f(\lambda) z_{1}^{p}(\lambda) d \lambda, \quad \forall t \in I
$$

Then $Y_{1}(t)$ is a positive and nondecreasing function on $I$ with $Y_{1}(0)=u_{0}^{p /(p+1)}$ and

$$
z_{1}(t) \leq Y_{1}(t)^{1 / p}
$$

Differentiating $Y_{1}(t)$ with respect to $t$, and using (2.6), (2.7) and (2.8), we get

$$
\begin{aligned}
\frac{d Y_{1}(t)}{d t} & \leq \frac{2 p}{p+1} \alpha^{\prime}(t) f(\alpha(t)) z_{1}^{p-1}(t) Y_{1}(t)+2 \alpha^{\prime}(t) f(\alpha(t)) z_{1}^{p}(\alpha(t)) \\
& \leq \frac{2 p}{p+1} \alpha^{\prime}(t) f(\alpha(t)) Y_{1}^{\frac{2 p-1}{p}}(t)+2 \alpha^{\prime}(t) f(\alpha(t)) Y_{1}(t), \quad \forall t \in I .
\end{aligned}
$$

From (2.9), we have

$$
Y_{1}^{\frac{1-2 p}{p}}(t) \frac{d Y_{1}(t)}{d t}-2 \alpha^{\prime}(t) f(\alpha(t)) Y_{1}^{\frac{1-p}{p}}(t) \leq \frac{2 p}{p+1} \alpha^{\prime}(t) f(\alpha(t)), \quad \forall t \in I .
$$

Let $S_{1}(t)=Y_{1}^{\frac{1-p}{p}}(t)$, then $S_{1}(0)=u_{0}^{\frac{1-p}{p+1}}$, from $(2.10)$ we obtain

$$
\frac{d S_{1}(t)}{d t}-2 \frac{1-p}{p} \alpha^{\prime}(t) f(\alpha(t)) S_{1}(t) \leq 2 \frac{1-p}{p+1} \alpha^{\prime}(t) f(\alpha(t)), \quad \forall t \in I .
$$

Consider the ordinary differential equation

$$
\left\{\begin{array}{l}
\frac{d S_{2}(t)}{d t}-2 \frac{1-p}{p} \alpha^{\prime}(t) f(\alpha(t)) S_{2}(t)=2 \frac{1-p}{p+1} \alpha^{\prime}(t) f(\alpha(t)), \quad \forall t \in I \\
S_{2}(0)=u_{0}^{\frac{1-p}{p+1}}
\end{array}\right.
$$

The solution of Equation (2.12) is

$$
\begin{aligned}
S_{2}(t)= & \exp \left(\int_{0}^{\alpha(t)} 2 \frac{1-p}{p} f(s) d s\right)\left(u_{0}^{\frac{1-p}{p+1}}\right. \\
& \left.+\int_{0}^{\alpha(t)} 2 \frac{1-p}{p+1} f(s) \exp \left(-\int_{0}^{s} 2 \frac{1-p}{p} f(\tau) d \tau\right) d s\right),
\end{aligned}
$$

for all $t \in I$. By (2.11), (2.12) and (2.13), we obtain

$$
Y_{1}(t)=S_{1}^{\frac{p}{1-p}}(t) \leq S_{2}^{\frac{p}{1-p}}(t)=\theta_{1}(t), \quad \forall t \in I
$$

where $\theta_{1}(t)$ as defined in (2.3). From (2.6) and (2.14), we have

$$
\frac{d z_{1}(t)}{d t} \leq \frac{2}{p+1} \alpha^{\prime}(t) f(\alpha(t)) \theta_{1}(t), \quad \forall t \in I .
$$


By taking $t=s$ in the above inequality and integrating it from 0 to $t$, we get

$$
u(t) \leq z_{1}(t) \leq u_{0}^{\frac{1}{p+1}}+\frac{2}{p+1} \int_{0}^{\alpha(t)} f(s) \theta_{1}\left(\alpha^{-1}(s)\right) d s, \quad \forall t \in I .
$$

The estimation (2.2) of the unknown function in the inequality (2.1) is obtained.

Theorem 2 Suppose $\alpha \in C^{1}(I, I)$ is increasing function with $\alpha(t) \leq t, \alpha(0)=0, \forall t \in I$. We assume that $u(t)$ and $f(t)$ are nonnegative real-valued continuous functions defined on $I$ and satisfy the inequality

$$
\begin{aligned}
u^{p+1}(t) \leq & u_{0}+\left(\int_{0}^{\alpha(t)} f(s) u^{p}(s) d s\right)^{2} \\
& +2 \int_{0}^{\alpha(t)} f(s) u^{p}(s)\left[u(s)+\int_{0}^{s} f(\lambda) u(\lambda) d \lambda\right] d s, \quad \forall t \in I
\end{aligned}
$$

where $u_{0}>0$ and $p \in(0,1)$ are constants. Then

$$
u(t) \leq u_{0}^{\frac{1}{p+1}}+\frac{2}{p+1} \int_{0}^{\alpha(t)} f(s) \theta_{2}\left(\alpha^{-1}(s)\right) d s, \quad \forall t \in I
$$

where

$$
\theta_{2}(t)=\beta_{2}(t)\left[u_{0}^{\frac{1-p}{p+1}}+\int_{0}^{\alpha(t)}(1-p) f(s) \exp \left(-\int_{0}^{s} \frac{(1-p)(p+3)}{p+1} f(\tau) d \tau\right) d s\right]^{\frac{1}{1-p}}
$$

and $\beta_{2}(t)=\exp \left(\int_{0}^{\alpha(t)} \frac{p+3}{p+1} f(s) d s\right)$, for all $t \in I$.

Proof Let $z_{2}^{p+1}(t)$ denote the function on the right-hand side of (2.15), which is a positive and nondecreasing function on $I$ with $z_{2}(0)=u_{0}^{\frac{1}{p+1}}$. Then $(2.15)$ is equivalent to

$$
u(t) \leq z_{2}(t), \quad u(\alpha(t)) \leq z_{2}(\alpha(t)), \quad \forall t \in I .
$$

Differentiating $z_{2}^{p+1}(t)$ with respect to $t$, using (2.18) we have

$$
\begin{aligned}
(p+1) z_{2}^{p}(t) \frac{d z_{2}(t)}{d t}= & 2 \alpha^{\prime}(t) f(\alpha(t)) u^{p}(\alpha(t)) \int_{0}^{\alpha(t)} f(s) u^{p}(s) d s \\
& +2 \alpha^{\prime}(t) f(\alpha(t)) u^{p}(\alpha(t))\left[u(\alpha(t))+\int_{0}^{\alpha(t)} f(\lambda) u(\lambda) d \lambda\right] \\
\leq & 2 \alpha^{\prime}(t) f(\alpha(t)) z_{2}^{p}(t)\left[z_{2}(t)+\int_{0}^{\alpha(t)} f(\lambda) z_{2}(\lambda) d \lambda\right. \\
& \left.+\int_{0}^{\alpha(t)} f(\lambda) z_{2}^{p}(\lambda) d \lambda\right], \quad \forall t \in I .
\end{aligned}
$$

Since $z_{2}^{p}(t)>0$, we have

$$
\frac{d z_{2}(t)}{d t} \leq \frac{2}{p+1} \alpha^{\prime}(t) f(\alpha(t)) Y_{2}(t), \quad \forall t \in I
$$


where

$$
Y_{2}(t):=z_{2}(t)+\int_{0}^{\alpha(t)} f(\lambda) z_{2}(\lambda) d \lambda+\int_{0}^{\alpha(t)} f(\lambda) z_{2}^{p}(\lambda) d \lambda, \quad \forall t \in I
$$

Then $Y_{2}(t)$ is a positive and nondecreasing function on $I$ with $Y_{2}(0)=z_{2}(0)=u_{0}^{1 /(p+1)}$ and

$$
z_{2}(t) \leq Y_{2}(t)
$$

Differentiating $Y_{2}(t)$ with respect to $t$, and using (2.20), (2.21) and (2.22), we get

$$
\begin{aligned}
\frac{d Y_{2}(t)}{d t} & \leq \frac{2}{p+1} \alpha^{\prime}(t) f(\alpha(t)) Y_{2}(t)+\alpha^{\prime}(t) f(\alpha(t)) z_{2}(\alpha(t))+\alpha^{\prime}(t) f(\alpha(t)) z_{2}^{p}(\alpha(t)) \\
& \leq \frac{p+3}{p+1} \alpha^{\prime}(t) f(\alpha(t)) Y_{2}(t)+\alpha^{\prime}(t) f(\alpha(t)) Y_{2}^{p}(t), \quad \forall t \in I .
\end{aligned}
$$

From (2.23), we have

$$
Y_{2}^{-p}(t) \frac{d Y_{2}(t)}{d t}-\frac{p+3}{p+1} \alpha^{\prime}(t) f(\alpha(t)) Y_{2}^{1-p}(t) \leq \alpha^{\prime}(t) f(\alpha(t)), \quad \forall t \in I
$$

Let $S_{3}(t)=Y_{2}^{1-p}(t)$, then $S_{3}(0)=u_{0}^{\frac{1-p}{p+1}}$, from $(2.24)$ we obtain

$$
\frac{d S_{3}(t)}{d t}-\frac{(1-p)(p+3)}{p+1} \alpha^{\prime}(t) f(\alpha(t)) S_{3}(t) \leq(1-p) \alpha^{\prime}(t) f(\alpha(t)), \quad \forall t \in I
$$

Consider the ordinary differential equation

$$
\left\{\begin{array}{l}
\frac{d S_{4}(t)}{d t}-\frac{(1-p)(p+3)}{p+1} \alpha^{\prime}(t) f(\alpha(t)) S_{4}(t)=(1-p) \alpha^{\prime}(t) f(\alpha(t)), \quad \forall t \in I \\
S_{4}(0)=u_{0}^{\frac{1-p}{p+1}}
\end{array}\right.
$$

The solution of Equation (2.26) is

$$
\begin{aligned}
S_{4}(t)= & \exp \left(\int_{0}^{\alpha(t)} \frac{(1-p)(p+3)}{p+1} f(s) d s\right)\left(u_{0}^{\frac{1-p}{p+1}}\right. \\
& \left.+\int_{0}^{\alpha(t)}(1-p) f(s) \exp \left(-\int_{0}^{s} \frac{(1-p)(p+3)}{p+1} f(\tau) d \tau\right) d s\right),
\end{aligned}
$$

for all $t \in I$. By (2.25), (2.26) and (2.27), we obtain

$$
Y_{2}(t)=S_{3}^{\frac{1}{1-p}}(t) \leq S_{4}^{\frac{1}{1-p}}(t)=\theta_{2}(t), \quad \forall t \in I
$$

where $\theta_{2}(t)$ as defined in (2.17). From (2.20) and (2.28), we have

$$
\frac{d z_{2}(t)}{d t} \leq \frac{2}{p+1} \alpha^{\prime}(t) f(\alpha(t)) \theta_{2}(t), \quad \forall t \in I .
$$


By taking $t=s$ in the above inequality and integrating it from 0 to $t$, we get

$$
u(t) \leq z_{2}(t) \leq u_{0}^{\frac{1}{p+1}}+\frac{2}{p+1} \int_{0}^{\alpha(t)} f(s) \theta_{2}\left(\alpha^{-1}(s)\right) d s, \quad \forall t \in I
$$

The estimation (2.16) of the unknown function in the inequality (2.15) is obtained.

Theorem 3 Suppose $\phi_{1}, \phi_{2}, \phi_{2} / \phi_{1}, \alpha \in C^{1}(I, I)$ are increasing functions with $\alpha(t) \leq t$, $\phi_{i}(t)>0, \forall t>0, i=1,2, \alpha(0)=0$. We assume that $u(t)$ and $f(t)$ are nonnegative real-valued continuous functions defined on I and satisfy the inequality

$$
\begin{aligned}
u(t) \leq & u_{0}+\left(\int_{0}^{\alpha(t)} f(s) \phi_{1}(u(s)) d s\right)^{2} \\
& +2 \int_{0}^{\alpha(t)} f(s) \phi_{1}(u(s))\left[u(s)+\int_{0}^{s} g(\lambda) \phi_{2}(u(\lambda)) d \lambda\right] d s, \quad \forall t \in I
\end{aligned}
$$

where $u_{0}>0$ is a constant. Then

$$
u(t) \leq \Phi_{1}^{-1}\left[\Phi_{2}^{-1}\left(\Phi_{2}\left(\Phi_{1}\left(u_{0}\right)+\int_{0}^{\alpha(t)} g(s) d s\right)+\int_{0}^{\alpha(t)} 2 f(s) d s\right)\right], \quad \forall t<T_{1}
$$

where

$$
\begin{aligned}
& \Phi_{1}(r):=\int_{1}^{r} \frac{d t}{\phi_{2}(t)}, \quad r>0, \\
& \Phi_{2}(r):=\int_{1}^{r} \frac{\phi_{2}\left(\Phi_{1}^{-1}(s)\right) d s}{\phi_{1}\left(\Phi_{1}^{-1}(s)\right)\left(\Phi_{1}^{-1}(s)+1\right)}, \quad r>0,
\end{aligned}
$$

and $T_{1}$ is the largest number such that

$$
\begin{aligned}
& \Phi_{2}\left(\Phi_{1}\left(u_{0}\right)+\int_{0}^{\alpha(t)} g(s) d s\right)+\int_{0}^{\alpha(t)} 2 f(s) d s \leq \int_{1}^{\infty} \frac{\phi_{2}\left(\Phi_{1}^{-1}(s)\right) d s}{\phi_{1}\left(\Phi_{1}^{-1}(s)\right)\left(\Phi_{1}^{-1}(s)+1\right)}, \\
& \Phi_{2}^{-1}\left(\Phi_{2}\left(\Phi_{1}\left(u_{0}\right)+\int_{0}^{\alpha(t)} g(s) d s\right)+\int_{0}^{\alpha(t)} 2 f(s) d s\right) \leq \int_{1}^{\infty} \frac{d t}{\phi_{2}(t)}
\end{aligned}
$$

for all $t \leq T_{1}$.

Proof Let $z_{3}(t)$ denote the function on the right-hand side of (2.29), which is a positive and nondecreasing function on $I$ with $z_{3}(0)=u_{0}$. Then (2.29) is equivalent to

$$
u(t) \leq z_{3}(t), \quad u(\alpha(t)) \leq z_{3}(t), \quad \forall t \in I .
$$


Differentiating $z_{3}(t)$ with respect to $t$, using (2.33) we have

$$
\begin{aligned}
\frac{d z_{3}(t)}{d t} \leq & 2 \alpha^{\prime}(t) f(\alpha(t)) \phi_{1}\left(z_{3}(t)\right) \int_{0}^{\alpha(t)} f(s) \phi_{1}\left(z_{3}(s)\right) d s \\
& +2 \alpha^{\prime}(t) f(\alpha(t)) \phi_{1}\left(z_{3}(t)\right)\left[z_{3}(t)+\int_{0}^{\alpha(t)} g(\lambda) \phi_{2}\left(z_{3}(\lambda)\right) d \lambda\right] d s \\
\leq & 2 \alpha^{\prime}(t) f(\alpha(t)) \phi_{1}\left(z_{3}(t)\right)\left[z_{3}(t)+\int_{0}^{\alpha(t)} f(s) \phi_{1}\left(z_{3}(s)\right) d s\right. \\
& \left.+\int_{0}^{\alpha(t)} g(\lambda) \phi_{2}\left(z_{3}(\lambda)\right) d \lambda\right], \quad \forall t \in I .
\end{aligned}
$$

Let

$$
Y_{3}(t):=z_{3}(t)+\int_{0}^{\alpha(t)} f(s) \phi_{1}\left(z_{3}(s)\right) d s+\int_{0}^{\alpha(t)} g(\lambda) \phi_{2}\left(z_{3}(\lambda)\right) d \lambda, \quad \forall t \in I .
$$

Then $Y_{3}(t)$ is a positive and nondecreasing function on $I$ with $Y_{3}(0)=z_{3}(0)=u_{0}$ and

$$
z_{3}(t) \leq Y_{3}(t)
$$

Differentiating $Y_{3}(t)$ with respect to $t$, and using (2.34), (2.35) and (2.36), we get

$$
\begin{aligned}
\frac{d Y_{3}(t)}{d t} \leq & 2 \alpha^{\prime}(t) f(\alpha(t)) \phi_{1}\left(z_{3}(t)\right) Y_{3}(t)+\alpha^{\prime}(t) f(\alpha(t)) \phi_{1}\left(z_{3}(t)\right) \\
& +\alpha^{\prime}(t) g(\alpha(t)) \phi_{2}\left(z_{3}(t)\right) \\
\leq & 2 \alpha^{\prime}(t) f(\alpha(t)) \phi_{1}\left(Y_{3}(t)\right)\left(Y_{3}(t)+1\right)+\alpha^{\prime}(t) g(\alpha(t)) \phi_{2}\left(Y_{3}(t)\right),
\end{aligned}
$$

for all $t \in I$. Since $\phi_{2}\left(Y_{3}(t)\right)>0, \forall t>0$, from (2.37) we have

$$
\frac{d Y_{3}(t)}{\phi_{2}\left(Y_{3}(t)\right)} \leq 2 \alpha^{\prime}(t) f(\alpha(t)) \frac{\phi_{1}\left(Y_{3}(t)\right)\left(Y_{3}(t)+1\right)}{\phi_{2}\left(Y_{3}(t)\right)}+\alpha^{\prime}(t) g(\alpha(t)), \quad \forall t \in I .
$$

By taking $t=s$ in the above inequality and integrating it from 0 to $t$, we get

$$
\begin{aligned}
\Phi_{1}\left(Y_{3}(t)\right) \leq & \Phi_{1}\left(Y_{3}(0)\right)+\int_{0}^{t} 2 \alpha^{\prime}(s) f(\alpha(s)) \frac{\phi_{1}\left(Y_{3}(s)\right)\left(Y_{3}(s)+1\right)}{\phi_{2}\left(Y_{3}(s)\right)} d s \\
& +\int_{0}^{t} \alpha^{\prime}(s) g(\alpha(s)) d s,
\end{aligned}
$$

for all $t \in I$, where $\Phi_{1}$ is defined by (2.31). From (2.38), we have

$$
\begin{aligned}
\Phi_{1}\left(Y_{3}(t)\right) \leq & \Phi_{1}\left(Y_{3}(0)\right)+\int_{0}^{T} \alpha^{\prime}(s) g(\alpha(s)) d s \\
& +\int_{0}^{t} 2 \alpha^{\prime}(s) f(\alpha(s)) \frac{\phi_{1}\left(Y_{3}(s)\right)\left(Y_{3}(s)+1\right)}{\phi_{2}\left(Y_{3}(s)\right)} d s
\end{aligned}
$$

for all $t<T$, where $0<T<T_{1}$ is chosen arbitrarily. Let $Y_{4}(t)$ denote the function on the right-hand side of (2.39), which is a positive and nondecreasing function on $I$ with $Y_{4}(0)=$ 
$\Phi_{1}\left(u_{0}\right)+\int_{0}^{T} \alpha^{\prime}(s) g(\alpha(s)) d s$ and

$$
Y_{3}(t) \leq \Phi_{1}^{-1}\left(Y_{4}(t)\right), \quad \forall t<T
$$

Differentiating $Y_{4}(t)$ with respect to $t$, using the hypothesis on $\phi_{2} / \phi_{1}$, from $(2.40)$ we have

$$
\begin{aligned}
\frac{d Y_{4}(t)}{d t} & \leq 2 \alpha^{\prime}(t) f(\alpha(t)) \frac{\phi_{1}\left(Y_{3}(t)\right)\left(Y_{3}(t)+1\right)}{\phi_{2}\left(Y_{3}(t)\right)} \\
& \leq 2 \alpha^{\prime}(t) f(\alpha(t)) \frac{\phi_{1}\left(\Phi_{1}^{-1}\left(Y_{4}(t)\right)\right)\left(\Phi_{1}^{-1}\left(Y_{4}(t)\right)+1\right)}{\phi_{2}\left(\Phi_{1}^{-1}\left(Y_{4}(t)\right)\right)}, \quad \forall t<T .
\end{aligned}
$$

By the definition of $\Phi_{2}$ in (2.32), from (2.41) we obtain

$$
\begin{aligned}
\Phi_{2}\left(Y_{4}(t)\right) & \leq \Phi_{2}\left(Y_{4}(0)\right)+\int_{0}^{t} 2 \alpha^{\prime}(s) f(\alpha(s)) d s \\
& \leq \Phi_{2}\left(\Phi_{1}\left(u_{0}\right)+\int_{0}^{\alpha(T)} g(s) d s\right)+\int_{0}^{\alpha(t)} 2 f(s) d s, \quad \forall t<T .
\end{aligned}
$$

Let $t=T$, from (2.42) we have

$$
\Phi_{2}\left(Y_{4}(T)\right) \leq \Phi_{2}\left(\Phi_{1}\left(u_{0}\right)+\int_{0}^{\alpha(T)} g(s) d s\right)+\int_{0}^{\alpha(T)} 2 f(s) d s
$$

Since $0<T<T_{1}$ is chosen arbitrarily, from (2.33), (2.36), (2.40) and (2.43), we have

$$
u(t) \leq \Phi_{1}^{-1}\left[\Phi_{2}^{-1}\left(\Phi_{2}\left(\Phi_{1}\left(u_{0}\right)+\int_{0}^{\alpha(t)} g(s) d s\right)+\int_{0}^{\alpha(t)} 2 f(s) d s\right)\right], \quad \forall t<T_{1}
$$

This proves (2.30).

\section{Application}

In this section, we apply our Theorem 3 to the following differential-integral equation

$$
\left\{\begin{array}{l}
\frac{d x(t)}{d t}=F(t, x(\alpha(t)))+H(t, x(\alpha(t))), \quad \forall t \in I, \\
x(0)=x_{0},
\end{array}\right.
$$

where $F \in C(I \times I, \mathbf{R}), H \in C\left(I^{3}, \mathbf{R}\right),\left|x_{0}\right|>0$ is a constant satisfying the following conditions

$$
\begin{aligned}
& |F(t, x(t))| \leq f^{2}(t) \phi_{1}^{2}(|x(t)|), \\
& |H(t, x(t))| \leq 2 f(t) \phi_{1}(|x(t)|)\left(|x(t)|+\int_{0}^{t} g(s) \phi_{2}(|x(s)|) d s\right),
\end{aligned}
$$

where $f, g$ is nonnegative real-valued continuous function defined on $I$.

Corollary 1 Consider the nonlinear system (3.1) and suppose that $F, H$ satisfy the conditions (3.2) and (3.3), and $\phi_{1}, \phi_{2}, \phi_{2} / \phi_{1}, \alpha \in C^{1}(I, I)$ are increasing functions with $\alpha(t) \leq t$, 
$\phi_{i}(t)>0, \forall t>0, i=1,2, \alpha(0)=0$. Then all solutions of Equation (3.1) exist on I and satisfy the following estimation

$$
|x(t)| \leq \Phi_{1}^{-1}\left[\Phi_{2}^{-1}\left(\Phi_{2}\left(\Phi_{1}\left(\left|x_{0}\right|\right)+\int_{0}^{\alpha(t)} \frac{g\left(\alpha^{-1}(s)\right)}{\alpha^{\prime}\left(\alpha^{-1}(s)\right)} d s\right)+\int_{0}^{\alpha(t)} 2 \frac{f\left(\alpha^{-1}(s)\right)}{\alpha^{\prime}\left(\alpha^{-1}(s)\right)} d s\right)\right],
$$

for all $t<T_{2}$, where

$$
\begin{aligned}
& \Phi_{1}(r):=\int_{1}^{r} \frac{d t}{\phi_{2}(t)}, \quad r>0, \\
& \Phi_{2}(r):=\int_{1}^{r} \frac{\phi_{2}\left(\Phi_{1}^{-1}(s)\right) d s}{\phi_{1}\left(\Phi_{1}^{-1}(s)\right)\left(\Phi_{1}^{-1}(s)+1\right)}, \quad r>0,
\end{aligned}
$$

and $T_{2}$ is the largest number such that

$$
\begin{aligned}
& \Phi_{2}\left(\Phi_{1}\left(\left|x_{0}\right|\right)+\int_{0}^{\alpha(t)} \frac{g\left(\alpha^{-1}(s)\right)}{\alpha^{\prime}\left(\alpha^{-1}(s)\right)} d s\right)+\int_{0}^{\alpha(t)} 2 \frac{f\left(\alpha^{-1}(s)\right)}{\alpha^{\prime}\left(\alpha^{-1}(s)\right)} d s \\
& \quad \leq \int_{1}^{\infty} \frac{\phi_{2}\left(\Phi_{1}^{-1}(s)\right) d s}{\phi_{1}\left(\Phi_{1}^{-1}(s)\right)\left(\Phi_{1}^{-1}(s)+1\right)}, \\
& \Phi_{2}^{-1}\left(\Phi_{2}\left(\Phi_{1}\left(\left|x_{0}\right|\right)+\int_{0}^{\alpha(t)} \frac{g\left(\alpha^{-1}(s)\right)}{\alpha^{\prime}\left(\alpha^{-1}(s)\right)} d s\right)+\int_{0}^{\alpha(t)} 2 \frac{f\left(\alpha^{-1}(s)\right)}{\alpha^{\prime}\left(\alpha^{-1}(s)\right)} d s\right) \leq \int_{1}^{\infty} \frac{d t}{\phi_{2}(t)}
\end{aligned}
$$

for all $t \leq T_{2}$.

Proof Integrating both sides of Equation (3.1) from 0 to $t$, we get

$$
x(t)=x_{0}+\int_{0}^{t} F(s, x(\alpha(s))) d s+\int_{0}^{t} H(s, x(\alpha(s))) d s, \quad \forall t \in I .
$$

Using the conditions (3.2) and (3.3), from (3.5) we obtain

$$
\begin{aligned}
|x(t)| \leq & \left|x_{0}\right|+\int_{0}^{t} f^{2}(s) \phi_{1}^{2}(|x(\alpha(s))|) d s \\
& +2 \int_{0}^{t} f(s) \phi_{1}(|x(\alpha(s))|)\left(|x(\alpha(s))|+\int_{0}^{s} g(\tau) \phi_{2}(|x(\alpha(\tau))|) d \tau\right) d s \\
\leq & \left|x_{0}\right|+\left(\int_{0}^{\alpha(t)} \frac{f\left(\alpha^{-1}(s)\right)}{\alpha^{\prime}\left(\alpha^{-1}(s)\right)} \phi_{1}(|x(s)|) d s\right)^{2} \\
& +2 \int_{0}^{\alpha(t)} \frac{f\left(\alpha^{-1}(s)\right)}{\alpha^{\prime}\left(\alpha^{-1}(s)\right)} \phi_{1}(|x(s)|)\left(|x(s)|+\int_{0}^{s} \frac{g\left(\alpha^{-1}(\tau)\right)}{\alpha^{\prime}\left(\alpha^{-1}(\tau)\right)} \phi_{2}(|x(\tau)|) d \tau\right) d s,
\end{aligned}
$$

for all $t \in I$. Applying Theorem 3 to (3.6), we get the estimation (3.4). This completes the proof of the Corollary 1. 


\section{Acknowledgement}

The author is very grateful to the editor and the referees for their helpful comments and valuable suggestions. This research was supported by National Natural Science Foundation of China (Project No. 11161018), Guangxi Natural Science Foundation (Project No. 0991265 and 2012GXNSFAA053009), Scientific Research Foundation of the Education Department of Guangxi Province of China (Project No. 201106LX599), and the Key Discipline of Applied Mathematics of Hechi University of China (200725).

Received: 12 March 2012 Accepted: 22 June 2012 Published: 5 July 2012

\section{References}

1. Gronwall, $\mathrm{TH}$ : Note on the derivatives with respect to a parameter of the solutions of a system of differential equations. Ann. Math. 20, 292-296 (1919). doi:10.2307/1967124

2. Bellman, R: The stability of solutions of linear differential equations. Duke Math. J. 10, 643-647 (1943). doi:10.1215/S0012-7094-43-01059-2

3. Lipovan, O: A retarded Gronwall-like inequality and its applications. J. Math. Anal. Appl. 252, 389-401 (2000). doi:10.1006/jmaa.2000.7085

4. Abdeldaim, A, Yakout, M: On some new integral inequalities of Gronwall-Bellman-Pachpatte type. Appl. Math. Comput. 217, 7887-7899 (2011). doi:10.1016/j.amc.2011.02.093

5. Agarwal, RP, Deng, S, Zhang, W: Generalization of a retarded Gronwall-like inequality and its applications. Appl. Math. Comput. 165, 599-612 (2005). doi:10.1016/j.amc.2004.04.067

6. Bihari, IA: A generalization of a lemma of Bellman and its application to uniqueness problem of differential equation. Acta Math. Acad. Sci. Hung. 7, 81-94 (1956). doi:10.1007/BF02022967

7. Pachpatte, BG: Inequalities for Differential and Integral Equations. Academic Press, London (1998)

8. Kim, YH: On some new integral inequalities for functions in one and two variables. Acta Math. Sin. 21, 423-434 (2005). doi:10.1007/s10114-004-0463-7

9. Cheung, WS: Some new nonlinear inequalities and applications to boundary value problems. Nonlinear Anal. 64 2112-2128 (2006). doi:10.1016/j.na.2005.08.009

10. Wang, WS: A generalized retarded Gronwall-like inequality in two variables and applications to BVP. Appl. Math. Comput. 191, 144-154 (2007). doi:10.1016/j.amc.2007.02.099

11. Wang, WS, Shen, C: On a generalized retarded integral inequality with two variables. J. Inequal. Appl. 2008, Article ID $518646(2008)$

12. Wang, WS, Li, Z, Li, Y, Huang, Y: Nonlinear retarded integral inequalities with two variables and applications. J. Inequal. Appl. 2010, Article ID 240790 (2010)

13. Wang, WS, Luo, RC, Li, Z: A new nonlinear retarded integral inequality and its application. J. Inequal. Appl. 2010, Article ID $462163(2010)$

doi:10.1186/1029-242X-2012-154

Cite this article as: Wang: A class of retarded nonlinear integral inequalities and its application in nonlinear differential-integral equation. Journal of Inequalities and Applications 2012 2012:154.

\section{Submit your manuscript to a SpringerOpen ${ }^{\circ}$ journal and benefit from:}

- Convenient online submission

Rigorous peer review

- Immediate publication on acceptance

- Open access: articles freely available online

- High visibility within the field

- Retaining the copyright to your article 